\title{
The many faces of VIP in neuroimmunology: a cytokine rather a neuropeptide?
}

\author{
DAVID POZO AND MARIO DELGADO*,1 \\ Department of Medical Biochemistry and Molecular Biology, University of Seville, Seville, Spain; and \\ *Institute of Parasitology and Biomedicine, CSIC, Granada, Spain
}

\begin{abstract}
Neuroimmunomodulation has experienced an explosive growth not only in basic research, but expanding to the point that prospective clinical research could be now a reality. A crucial factor for the functioning of this intimate bidirectional network was the demonstration that the immune and neuroendocrine systems speak a mutual biochemical language. This implies 1) production of neuroendocrine hormones and neuropeptides by immune cells and of cytokines by neuroendocrine cells; 2) evidence for shared receptors on cells of the immune and neuroendocrine systems; 3) effect of neuroendocrine mediators on immune functions; and 4) effect of cytokines on the neuroendocrine system. This reduces traditional differences between neurotransmitters, hormones, and immune mediators and raises the following question: what can we now regard as immune or neuroendocrine? Vasoactive intestinal peptide (VIP) is one example of this paradigm. VIP has traditionally been classified as a neuropeptide/neurotransmitter based in its capacity to mediate and regulate neuronal functions. Recent work has demonstrated that VIP is produced by $T$ cells, especially $\mathrm{Th} 2$ cells, and that through specific receptors it exerts immunological functions typically ascribed to Th2 cytokines in nervous and immune systems. Here, we postulate that instead of a neuropeptide, VIP could be fully considered a type 2 cytokine with a key role in neuroimmunology.-Pozo, D., Delgado, M. The many faces of VIP in neuroimmunology: a cytokine rather a neuropeptide? FASEB J. 18, 1325-1334 (2004)
\end{abstract}

Key Words: immune response $\cdot$ autoimmunity $\cdot$ Th cells $\cdot$ vasoactive intestinal peptide

FOR MANY YEARS immunologists have considered an immune response to be a direct consequence of, and solely determined by, antigenic stimulation of an autonomous immune system, clearly pointing out the key molecular questions for experimental investigation. However, over the past two decades, we have come to realize that the immune system is regulated by the central nervous system (CNS) either directly or by way of the neuroendocrine axis (i.e., the hypothalamicpituitary-adrenal system, HPA axis) or the autonomic nervous system. The importance of these interactions is underscored by a demonstration that immune and nervous interactions, including neuropeptides, cyto- kines, and specific immunomodulatory enzymes, have been well conserved during the course of evolution. Neuroimmunology is now sufficiently mature to begin to consider organization, workings, behavior, and applications. A crucial factor for this intimate bidirectional network to function was a demonstration that the immune and neuroendocrine systems speak a mutual biochemical language (Fig. 1). This reduces traditional differences between neurotransmitters, hormones, and immune mediators and raises the question of what can actually be considered as immune or neuroendocrine? More important, the final outcome will most likely lead to new unifying ideas and paradigms. The present developmental stage in neuroimmunology in general, and in VIP research in particular, can be characterized as a transition from analysis (the reduction of observations to key molecules) to synthesis (an integration of the parts into a whole). The difficulty in answering this question starts when we try to define the terms neuropeptide and cytokine exactly. De Wied and colleagues coined the term neuropeptide in 1974 by defining it as endogenous substances synthesized by nerve cells and involved in nervous system functions (1). However, whereas Kastin and Strand (2, 3) define a neuropeptide as any peptide (independent of its cell source) that has an action on the nervous system, Iversen defines it as any peptide secreted from a neuron (4). Although de Wied's definition appears to correspond to that accepted by most neuroscientists, the discrepancy is served.

Cytokines, on the other hand, are defined as small regulator proteins that are expressed in and secreted from immune cells and control survival, growth, differentiation as well as effector functions of their target cells. Originally identified as being important in inflammatory processes, the development and maintenance of immune response, and for hematopoiesis, it is now becoming evident that cytokines are involved at least to some extent in most, if not in all, physiological processes. Progress with the identification of new cytokine molecules is especially fast moving and new molecules are discovered with alarming frequency. Although cy-

\footnotetext{
${ }^{1}$ Correspondence: Institute of Parasitology and Biomedicine, CSIC, Avd. Conocimiento, Granada, 18100 Spain. E-mail: mdelgado@ipb.csic.es doi: 10.1096/fj.03-1440hyp
} 


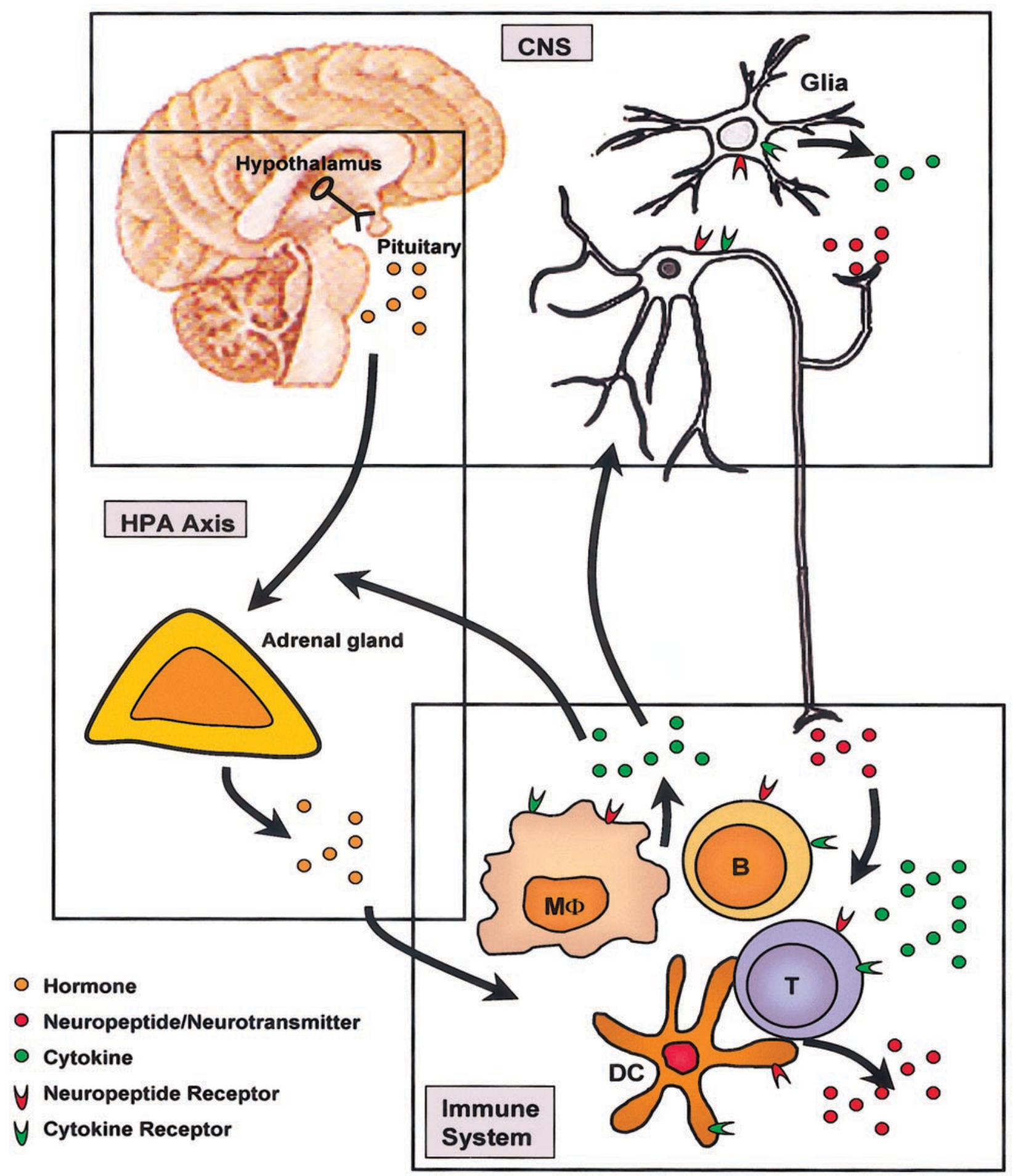

Figure 1. The bidirectional interactions between the neuroendocrine and immune systems are mediated by anatomical connections and mediators released and recognized by both systems, implying 1 ) neuroendocrine hormones and neuropeptides produced by immune cells and cytokines by neuroendocrine cells; 2) common receptors expressed on cells of the immune and neuroendocrine systems; 3) effects of neuroendocrine mediators on immune functions; and 4) effects of cytokines on the neuroendocrine system.

tokines were considered to be a "family," this is a functional rather than a structural concept, as these proteins are not all chemically related. Cytokines encompass those families of regulators variously known as growth factors, colony-stimulating factors, interleukins, lymphokines, monokines, and interferons.
The confusing nomenclature of cytokines has arisen as several different streams of investigation led to the discovery of different cytokines and, for example, molecules initially considered growth factors were later reclassified as cytokines. Conversely, interleukins were defined in 1981 as molecules made by leukocytes that 
acted on leukocytes. Subsequent research has revealed that some of these molecules are also made by and act on nonleukocytes, and the nomenclature has stuck. Despite overwhelming evidence for key immunomodulatory roles, the classical endocrine hormones have not always enjoyed a good press, and modern textbooks of immunology give scant attention to these compounds, mainly because their general properties are different from those of cytokines (Table 1). Nevertheless, there are no sharp boundaries that distinguish cytokines from other regulatory proteins. Some neuropeptides are between these boundaries.

In this sense, VIP is a paradigmatic polypeptide that has evolved from little more than a mere neuropeptide/hormone to a novel agent for modifying immune function and a possible a cytokine-like molecule. First identified by Said and Mutt in the late 1960s, VIP was originally isolated as a vasodilator and hypotensive peptide $(5,6)$. Later, its biochemistry was elucidated and VIP's signature features as a neuropeptide/neurotransmitter were consolidated, as a neuromodulator in many organs and tissues, including heart, lung, thyroid gland, kidney, immune system, urinary tract, and genital organs (7). The widespread distribution of VIP is correlated with its involvement in a wide variety of biological processes, from systemic vasodilatation, with recently reported potential use as a new drug for treatment of primary pulmonary hypertension (8), to core clock functions, being the first neuropeptide reported to be important for maintaining stable circadian clock function (9). It did not take long for these insights to permeate the field of immunology, from which surprising new attributes for VIP have been found over the last few years. VIP is rapidly transforming into something more than a mere hormone/ neuropeptide, evolving scientifically from a neuropeptide to a novel agent for modifying immune function and a possible a cytokine-like molecule. VIP research has engaged many physiologists, molecular biologists, biochemists, endocrinologists, and pharmacologists, and VIP presents a paradigm to explore mutual interactions between neural and neuroendocrine links in health and disease.

During the last 5 years, the molecular machinery accountable for the VIP-mediated anti-inflammatory effects (10-12) has been unraveled. VIP is by far the first neuropeptide able to drive Th cell differentiation. This is the framework for integrating the effects of VIP on innate and adaptive immunity and for developing alternative strategies against chronic inflammatory diseases and autoimmune disorders (13). Anti-inflammatory effects have recently been extended to those effects through chemokine receptor function (14). Thus, VIP may play an important role in problems related to complexity and self-organization in a manner similar to cytokines. In the next paragraphs, we highlight the principal issues that have arisen in this area and offer a final reasoned outcome: that VIP should be considered as a Th2 cytokine.

The key characteristics that a certain molecule must possess in order to be considered a Th2 cytokine are to 1) be produced by Th2 cells in response to a specific antigen stimulation; 2) stimulate and inhibit Th2 and Th1 function/generation, respectively; 3) mediate autocrine/paracrine actions involving the expression of specific receptor in neighboring cells, and 4) act in a pleiotropic and redundant way, inducing a Th2 biological action (reducing the cellular-mediated inflammatory response, favoring the humoral response). VIP combines all of these.

\section{VIP is produced by Th2 cells}

It was recently that VIP was found to be released by lymphoid innervation in close proximity to immune cells and produced by most lymphocyte types (11). However, VIP has been found to be specifically synthesized and secreted by Th2 cells after specific antigen stimulation (15). The physiological relevance of VIP production by Th2 effectors might reside in the fact that endogenous and exogenous VIP both promote Th2-type responses in vivo, implicating VIP in the Th2 amplification loop.

\section{VIP promotes Th2-type immune responses}

The adaptive immune response against a specific antigen is initiated by the cellular contact between an antigen-presenting cell (APC) and a naive T cell bear-

TABLE 1. General differences between cytokines and endocrine hormones

\begin{tabular}{|c|c|c|}
\hline Property & Cytokines & Hormones \\
\hline Sites of production & Many & Few \\
\hline Cell targets & Few & Many \\
\hline \multirow[t]{4}{*}{ Biological role } & Immunity & Homeostasis \\
\hline & Inflammation & \\
\hline & Fighting infection & \\
\hline & Tissue repair & \\
\hline Redundancy & High & Low \\
\hline Pleiotropic action & High & Low \\
\hline Circulatory levels & Very low & Moderate/high \\
\hline Influence range & Local: autocrine/paracrine & Systemic: widespread \\
\hline Inducers & External insults & Physiologic variation \\
\hline
\end{tabular}


ing the appropriate $\mathrm{T}$ cell receptor (TCR). Stimulatory (MHC-II/Ag on the APC, TCR on the T cell) and costimulatory (B7.1/B7.2 and CD40 on the APC and CD28 and CD40L on the T cell) interactions are both required for activation of naive $\mathrm{T}$ cells. Upon activation, antigen-specific T cells express high-affinity IL-2 receptors and proliferate in response to autocrine or paracrine IL-2. After antigenic stimulation and proliferation, CD4 $\mathrm{T}$ cells differentiate into Th1 and Th2 effector cells, characterized by specific cytokine profiles and functions. Th1 cells secrete cytokines (IL-2, IFN $\gamma$, and TNF $\beta$ ) critical for generation of a cellular immune response, thereby activating macrophages, inducing delayed-type hypersensitivity responses, and stimulating IgG2a antibody production by B cells in mice. Th2 cells produce IL-4, IL-5, and IL-10, which are critical for IgG1 and IgE synthesis and immunity against helminthic parasites; they increase of allergic reactions and inhibit macrophage activation and antigen presentation, down-regulating the cellular immune responses (Fig. 2). Determining factors for differentiation into Th1 or Th2 effectors include the nature of the APCs, the nature and amount of antigen, the genetic background of the host, and particularly the cytokine microenvironment. Recent studies clearly demonstrate that VIP acts as an important endogenous $\mathrm{T}$ helper differentiating factor, promoting Th2-type and inhibiting Th1-type responses in vivo and in vitro. Indeed, macrophages and dendritic cells (DC) treated in vitro with VIP gain the ability to induce Th2-type cytokines (IL-4, IL-5) and inhibit Th1-type cytokines (IFN $\gamma$, IL-2) in antigen-primed CD4 T cells. In agreement with in vitro results, in vivo administration of VIP in immunized mice results in a decreased number in Th1 cells and an increased number of Th2 cells $(16,17)$. In another different approach, two experimental animal models clearly show the Th2 bias mediated by VIP (18, 19). The research team led by Goetzl used genetically modified mice to overcome the low potency of VIP receptor antagonists. The autocrine/paracrine role of VIP as a Th2 cytokine-like molecule is possible by fully functional expression of VIP receptors in the immune system. The first reports of VIP receptors in the immune system were claimed in human peripheral blood lymphocytes in the early 1980s (20). Receptors for VIP and the related molecule PACAP belong to group II of the G-protein-coupled receptors. So far, three receptors that display high affinity for VIP and PACAP have been cloned and, according to the established IUPHAR nomenclature, are named $\mathrm{VPAC}_{1}, \mathrm{VPAC}_{2}$ (high affinity for both VIP and PACAP), and PAC $_{1}$ (selective affinity for PACAP). The best-characterized effect after the engagement of VPAC receptors is the accumulation of intracellular cAMP and the activation of PKA through stimulation of the $\alpha$ subunits of Gs proteins. Expression of genes encoding VPAC receptors are relatively well characterized in the immune system, although the molecular mechanisms that drive their transcription are poorly understood. $\mathrm{VPAC}_{2}$ expression on $\mathrm{T}$ cells is induced only after TCR stimulation or exposure to particular cytokines whereas $\mathrm{VPAC}_{1}$ mRNA is constitutively expressed. Thus, Goetzl and co-workers engineered a $\mathrm{T}$ cell-targeted mouse line in which $\mathrm{VPAC}_{2}$ is constitutively expressed on $\mathrm{CD}^{+} \mathrm{T}$ cells at levels characteristic of those in normal cells after maximal TCR stimulation (18) and a counterpart $\mathrm{VPAC}_{2}{ }^{-/-}$ knockout mouse line (19). The two models showed opposite immune phenotypes - the former toward a Th2 profile and the latter toward a Th1 profile. Consequently, $\mathrm{VPAC}_{2}$ null mice have increased cutaneous delayed-type hypersensitivity and markedly decreased cutaneous immediate-type hypersensitive. Therefore, the major differences between effector $\mathrm{T}$ cell phenotypes of these mouse models are clearly dependent on $\mathrm{T}$ cell VIP receptors and cytokines and generally agree as to opposite skewing of the Th1/Th2 cytokine ratio. A definitive confirmation of the role of endogenous, Th2-derived VIP in maintaining the Th2 bias recently came from studies by Voice et al., in which elimination of VIP from TCR-stimulated T cells from VPAC ${ }_{2}$ transgenic mice with VIPase IgG resulted in a readjustment of the Th1/Th2 balance, decreasing secretion of IL-4 and IL-10 and increasing the production of IFN $\gamma$ (21). Several nonexcluding mechanisms could contribute to the Th2 bias by VIP. The spectrum of VIP effects leading to Th2 cell predominance includes action at the level of Th1/Th2 generation, either directly or through actions on APCs and/or at the level of the already generated effectors, by selectively promoting Th2 proliferation, survival, or accumulation (Fig. 2).

Data from studies of $\mathrm{VPAC}_{2}$ transgenic mice highlight the fact that endogenous VIP recognized by $\mathrm{CD} 4^{+}$ $\mathrm{T}$ cells is a key factor in the final $\mathrm{Th}$ phenotype outcome (21). Therefore, we should start thinking about how to explain the apparently contradictory results from a new standpoint in which VIP is regarded as a cytokine. Thus, VIP could act at different operational levels. In vivo animal disease models can show us how to determine whether VIP is or is not set as a regulatory cytokine and at which operational level in a given disease compared with a physiological scenario. These data will then provide us with the insight needed to define whether therapeutic application should be effective or not.

\section{VIP is an endogenous anti-inflammatory factor}

Successful elimination of pathogens requires the initiation and participation of innate and adaptive immunity. However, once the pathogen is eliminated, cells participating in innate and adaptive immunity must be deactivated or eliminated in order to re-establish immune homeostasis. Uncontrolled activation of the immune system leads to serious consequences for the host such as tissue destruction and organ failure, even death. In addition to apoptosis of activated immune cells, several endogenous agents such as anti-inflammatory cytokines, lipid mediators, and glucocorticoids down-regulate inflammatory processes. Recently, VIP was added to the list of endogenous anti-inflammatory 
agents. This is a general prerequisite for a Th2 cytokine. Thus, VIP has been used or suggested for the development of therapies for several disorders (22), but its role as a Th2 cytokine makes it an attractive asset for studying inflammatory and/or Th1-type autoimmune diseases. The clinical implications of VIP research include septic shock, rheumatoid arthritis, Crohn's disease, Parkinson's disease (PD), and brain trauma. Some of the mechanisms involved emphasize the cytokine-related nature of VIP as striking parallelisms with typical Th2 cytokines such as IL-4, IL-10, or IL-13 become apparent.

It is generally recognized that the severe pathological consequences of the septic shock syndrome give rise to a hyperactive and an out-of-balance network of endogenous proinflammatory cytokines including TNF $\alpha$, IL12 , IL-6, and IFN $\gamma$ (23). The overproduction of inflammatory cytokines generates systemic activation, which affects vascular permeability and cardiac function, induces metabolic changes that can lead to tissue necrosis, and eventually to multiple organ failure and death. Since VIP inhibits the production of proinflammatory macrophage-derived cytokines in vivo and in vitro, it was expected to protect against endotoxemia. Indeed, VIP administration protects against high lethal endotoxemia in a murine model for septic shock syndrome (24). Endotoxemic animals suffer from generalized intravascular coagulation with multiple organ failure as evidenced by severe congestion, hemorrhage, hyperemia, fibrin deposits, edema, thrombosis, and massive accumulation of leukocytes in lungs and the intestinal tract, as well as severe congestion of the medullar sinusoids in the spleen, segmental ischemia of the bowel with regions of hemorrhage or necrosis, and an infracted cecum. In contrast, VIP-treated individuals did not present any of the histopathological alterations associated with septic shock such as disseminated intravascular coagulation, leukocyte infiltration and inflammation in various organs, mesenteric ischemia, and acute tubular necrosis in the kidneys (24). VIP acts presumably by down-regulating proinflammatory mediators such as TNF $\alpha$, IFN $\gamma$, IL-6, IL-12, and NO and up-regulating production of IL-10 (25). Furthermore, the inhibitory effect of VIP on the production of proinflammatory chemokines is crucial in its preventive action on endotoxemia (26), because transmigration of inflammatory cells to target inflamed organs is one of the Initial events. It has recently been reported that VIP inhibits cell responses to chemokines in monocytes and $\mathrm{CD}^{+} \mathrm{T}$ cells by trans-deactivating chemokine receptors, a mechanism involving chemokine receptor phosphorylation (14). Other studies (27) have suggested that the protective effect of VIP on septic shock syndrome is mediated through the regulation of serum levels of hormones such as adrenaline and cortisol, which control hemodynamic constants, important events in the pathology of sepsis.

VIP has also emerged as an attractive candidate for the treatment of arthritis. Two recent reports $(13,28)$ using the animal model of collagen-induced arthritis
(CIA) have demonstrated that treatment of arthritic mice with VIP decreases the frequency, delays the onset, and reduces the severity of the disease. The therapeutic effect of VIP on arthritis is associated with a striking reduction of the two deleterious components of the disease: the autoimmune and inflammatory response. VIP reduces the titter of autoreactive anticollagen antibodies (particularly IgG2a antibodies) in response to a reduction in the collagen-specific $\mathrm{T}$ cell response, accompanied by a Th2 hallmark reduction in IFN $\gamma$ production and an increase in IL-4. There are possible side effects to the chronic administration of VIP, including gastrointestinal effects and generalized immunosuppression, but such adverse effects were observed short-term in the experimental CIA model. Regarding its effect in joint inflammation, VIP strongly reduces the inflammatory response during arthritis development by down-regulating production of several proinflammatory agents in the inflamed joints and synovial cells, including TNF $\alpha$, IL-6, IL-1 $\beta$, iNOS, IL-12, and IL-18, as well as various chemokines (RANTES, MCP-1, MIP-1 $\alpha$, MIP-1 $\beta$, MIP-2) reported to play a role in inflammation and in the development of arthritic responses (29). VIP levels are specifically increased in serum and joints of arthritic mice during disease development (30), demonstrating that endogenous neuroimmune factors act as natural anti-arthritic agents activated in response to autoimmune/inflammatory conditions to counterbalance the effects of inflammatory mediators.

Although its etiology is unknown, Crohn's disease has been shown to be marked by an exaggerated gut-associated, lymphoid tissue-developed immune response, giving rise to a prolonged severe inflamed intestinal mucosa characterized by uncontrolled production of proinflammatory cytokines and by oligoclonal expansion and activation of $\mathrm{CD}^{+} \mathrm{T}$ cells, specifically associated with a Th1 response (31). In a recent report, Abad and co-workers (32) used the established murine model of Crohn's disease induced by intrarectal administration of trinitrobenzene sulfonic acid (TNBS) to demonstrate that treatment with VIP reduced the clinical and histopathologic severity of TNBS-induced colitis, abrogating body weight loss, diarrhea, and intestinal inflammation. The therapeutic effects of VIP occurred in all phases of the disease (early, acute, and chronic). In the early acute phase of bowel inflammation there is an overlapping of innate and adaptive immune responses, with multiple mediators involved, such as chemokines and cytokines. VIP significantly reduced the inflammatory response by down-regulating the production of different proinflammatory mediators involved in local and systemic damage, such as TNF $\alpha$, IL-1 $\beta$, IL-6, IL-12, MIP-1 $\alpha$, MCP-1, MIP-2, and KC. This broad spectrum of action is related to a decrease in colitis-associated inflammation and the infiltration of neutrophils, macrophages, and $\mathrm{CD} 4^{+} \mathrm{T}$ cells in the lamina propria. Treatment with VIP partially prevented TNBS-induced generation of $\mathrm{CD} 4^{+} \mathrm{T}$ cells and of Th1 responses. From a therapeutic point of 

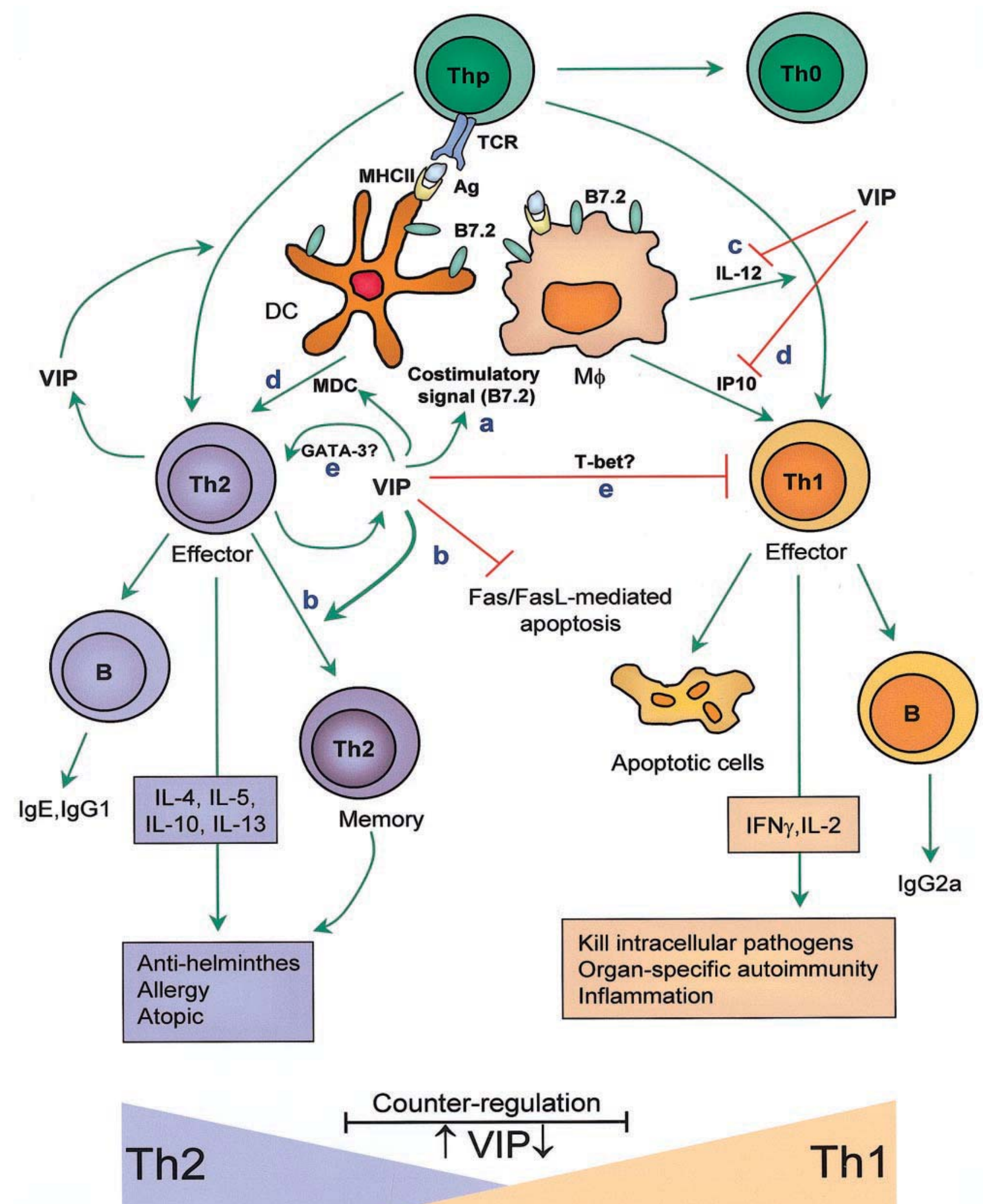

Figure 2. Effects of VIP on $\mathrm{CD} 4^{+} \mathrm{T}$ cell activation and differentiation. The same Th precursor (Thp) cell can differentiate into Th0, Th1, or Th2 cells depending primarily on the cytokine microenvironment provided by DC and other antigen-presenting cells (APCs): IL-12 induces Th1 cells, IL-4 promotes Th2 cells, whereas Th0 cells differentiate under neutral conditions with optional IL-2. In addition, VIP released from antigen (Ag) -stimulated Th2 cells drives Th differentiation/generation acting on CD4 T cells and APCs. a) Action on APCs: differentiation into Th2 effectors depends on the expression of the membrane-bound costimulating factors B7 to a much larger degree than differentiation into Th1 effectors. VIP induces B7.2 expression on resting macrophages $(\mathrm{M} \phi)$ and DCs and promotes Th2-type responses. b) Action on Th1/Th2 effectors: the majority of Th1 and Th2 effectors are eliminated through Fas/FasL-mediated apoptosis (AICD, antigen-induced cell death), with a small number 
view, it is important to point out that VIP reduced disease severity even when given after the onset of the disease. Therefore, VIP could represent a possible multistep therapeutic agent for Crohn's disease.

In several neurodegenerative disorders, including PD, Alzheimer's disease, multiple sclerosis, and AIDS dementia, neuronal cell death occurs subsequent to inflammatory responses, mediated at least partially by activated microglia (33). Proinflammatory cytokines are known to participate in mitochondrial impairment and oxidative stress; therefore, an inflammatory response may serve as an integral feature of the mechanistic underpinning related to PD pathogenesis. Because VIP has been shown to act as a "microgliadeactivating factor" under inflammatory conditions and to prevent inflammation-induced neurodegeneration (34), this peptide emerges as a plausible candidate for neuroprotection under inflammatory conditions, including PD. VIP prevents MPTP-induced activation of microglia and expression of the cytotoxic mediators iNOS, IL-1 $\beta$, and TNF $\alpha$, and NADPH-oxidase. These findings indicate important future directions, including a possible therapeutic role of VIP in brain disorders such as PD itself or multiple sclerosis, Alzheimer's diseases, and AIDS dementia, where the inflammatory response plays a major role. Recently, VIP cytokine-like behavior has demonstrated a potential therapeutic effect of VIP in brain traumas, where an increased level of proinflammatory cytokines is a normal and early feature of the CNS response to trauma (35); it remains controversial, however, whether inflammation in the injured CNS serves a beneficial or detrimental purpose (36). In a model of adult mice stab wound brain trauma in the periventricular area, VIP has been shown to significantly reduce all pathological hallmarks that follow brain injury: dramatic neurodegeneration, recruitment of mononuclear phagocytes, microglia activation, and increased production of TNF $\alpha$ and IL-1 $\beta$ (37). A direct link between VIP and the response to neuronal insults has been reported. In this case, VIP provides neuronal defense by inducing the synthesis and secretion of neuroprotective proteins from astrocytes (38).

VIP modulates the function of inflammatory cells through specific G-protein-coupled receptors (Fig. 3); extensive literature has been published on VIP receptors (39). We shall focus here on some of the questions that remain to be addressed as well as some of the hypotheses currently being scrutinized in our laboratory. Recent advances in molecular and cell biology enabling the cloning, expression, and mutagenesis of VIP receptors have prompted an explosion of knowledge in the field, facilitating the discovery of new classes of regulatory proteins and providing a basis and means for manipulating receptor function. Combined functional information from early stages in VIP receptor research showed several common features such as a strong coupling to the activation of adenylyl cyclase and the ability to interact in a species-specific manner with related peptides such as secretin, helodermin, growth hormone-releasing factor, or peptide histidine isoleucineamide. Some of these peptides were encoded within the VIP precursor polypeptide mRNA as additional biologically active peptides acting most likely through VIP receptors (as mentioned above; $\mathrm{VPAC}_{1}$, $\mathrm{VPAC}_{2}$, and $\mathrm{PAC}_{1}$ ) instead of different and specific sets of receptors. An open question still to be determined is to what extent these peptides have immunomodulatory capabilities and whether this degeneracy could regulate the effects mediated by the VIP itself. There are no data on possible differential post-translational processing under different immune conditions to account for putative fine-tuning mechanisms of counter regulation exploiting the "less-than-discriminate" nature of ligandreceptor interactions. From a pharmacological point of view, these natural peptides structurally related to VIP have a limited utility. An important point in establishing the role of VPAC receptors was the development during the past few years of selective agonists and antagonists. Sequence alignment of PACAP, VIP, [K15, R16, L27] $\mathrm{VIP}_{(1-7)} / \mathrm{GRF}_{(8-27)}$, Ro 25-1553, and Ro 251392 was recently analyzed to identify $\mathrm{VPAC}_{2}$ selective determinants by saturation recombinant mutagenesis, leading to the production of a highly selective and potent $\mathrm{VPAC}_{2}$ agonist as a recombinant peptide (40). The strategy followed by the above-cited authors overcame important technical issues such as the absence of exogenous amino acids without losing stability and analysis of a large number of peptides and provides a feasible method of industrial manufacture. It has recently been shown that immunological tolerance to VIP can be broken (41); thus, further regulation of VIP by autoantibodies is undetermined. The possible presence of autoreactive $\mathrm{T}$ clones against VIP could lead to a new ways of immunointervention by $\mathrm{T}$ cell vaccination.

Localization of VPAC receptors and its consequences on immunocompetent cells would lead to new paradigms of VIP's mechanism of action. We are only beginning to unravel the functional implication that G-protein-coupled receptor homo- and heterodimerization represent. Future research in VPAC receptor dimerization could be critical for novel drug design in the context of the immune system. After binding to their specific receptors, chemokines, as occurs for other GPCRs, induce receptor dimerization. Bioinformatic analysis shows that some dy-

surviving as memory T cells. VIP promotes the preferential survival of Th2 vs. Th1 effectors. $c$ ) Action on activated macrophages: VIP inhibits IL-12 production by activated macrophages, reducing differentiation into Th1 effectors. $d$ ) Action on Th1/Th2specific chemokines: IP10 and MDC are DC/macrophage-derived chemokines that specifically attract Th1 and Th2 cells, respectively. VIP inhibits and stimulates IP-10 and MDC production, respectively, and favors Th2 vs. Th1 chemoattraction to the antigen presentation site. $e$ ) The possibility exists (indicated by a question mark) that VIPs act directly on CD $4^{+} \mathrm{T}$ cells and bias Th1/Th2 differentiation in favor of Th2 by promoting the expression of Th2 transcription factors (c-Maf, JunB, GATA-3) and inhibiting the expression of T-bet (the Th1 master transcription factor). 

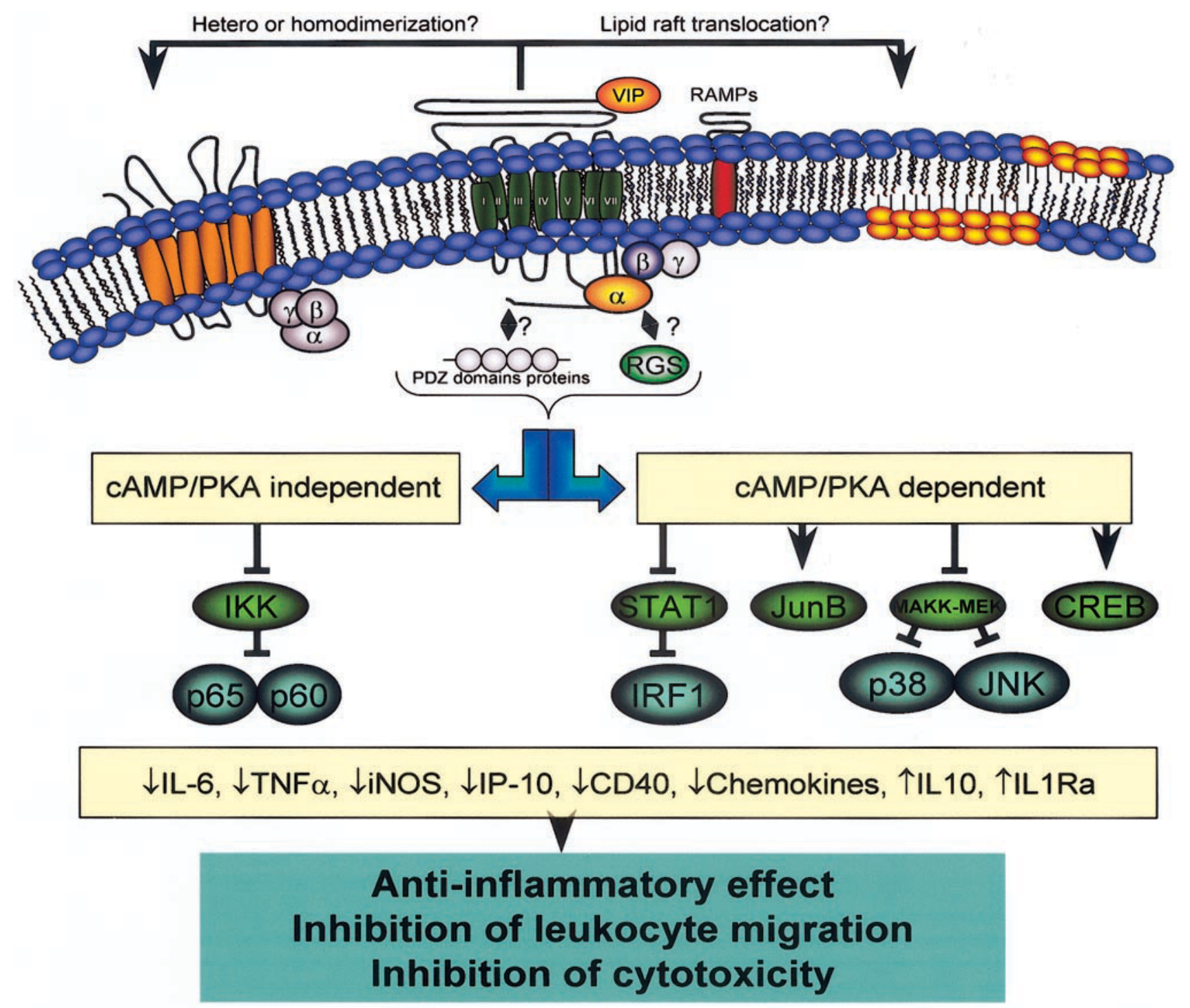

Figure 3. VPAC receptors on immune system couple to 1) stimulation of adenylyl cyclase triggering a protein kinase A (PKA)-cAMP transduction pathway, and 2) activation of phospholipase C (PLC) and phospholipase D (PLD). VPAC receptors induce responses by activating transduction systems that involve different G-proteins, with $\mathrm{G} \alpha$ as the best characterized in different tissues and cell lines expressing recombinant receptors. Other G-proteins that have been shown to be coupled to VPAC receptors belong to $\mathrm{Gi} / \mathrm{Go}$ and $\mathrm{Gq}$ families $(42,43)$. Other VPAC partners different from G-proteins have been reported such as the small G-protein ADP-ribosylation factor (ARF) or receptor activity-modifying proteins (RAMP), resulting in alterations of receptor phenotype or pharmacological profile $(44,45)$. These are not yet documented on immunocompetent cells. New binding partners are being investigated by us as regulators of G-protein signaling (RGS). RGS proteins are important acting as tightly regulated integrators of G-protein signaling and as scaffolds to assemble signaling complexes. PDZ motif-containing proteins are also scaffold proteins with regulatory functions. $\mathrm{VPAC}_{1}$ and probably $\mathrm{VPAC}_{2}$ contain a typical binding site for PDZ domains. The physiological significance of PDZ-VPAC receptor interaction on immunocompetent cells is an open question. It is interesting to hypothesize on the physical compartmentalization of VPAC receptors and the consequences in signal transduction. Lipid rafts function in the initiation of signaling in a variety of immune cells and in the control of signaling by coreceptors during development and differentiation and of infection. In this sense, lipid raft localization of VPAC receptors is under current scrutiny.

namic interactions between VPAC receptors and chemokine receptors are possible.

\section{CONCLUSIONS}

Could VIP be considered a type 2 cytokine? The term "cytokine" defines a protein secreted by leukocytes (and other cell types) in response to tissue injury or repair, acting predominantly in a local, paracrine, or autocrine manner, with pleiotropic actions, including effects on immune cells and modulation of the inflammatory response. If, in addition, such a protein is produced by Th2 cells, it affects the Th1/Th2 balance, enhancing Th2 and reducing Th1 generation/function, resulting in a generalized anti-inflammatory, antihelminthic, and atopic function, it is then included in 
the group of type 2 cytokines. The findings shown here indicate that VIP shares these characteristics.

It is clear that VIP is pleiotropic to an extreme. Further research in our functional genomics era will address how many genes VIP regulates at a molecular level. These genes will probably be more or less pleiotropic themselves. Therefore, a single molecule like VIP is pleiotropic in a well-known cytokine manner at the most basic causal level. Moreover, the functional overlap between VIP and different immune-related molecules presents another well-known cytokine characteristic, that of redundancy. VIP and other Th2 cytokines will only partially overlap in their effects; each molecule produces effects that others do not. This degenerate redundancy (partially overlapping pleiotropisms) is a hallmark of cytokines.

In any case, whether VIP is considered a neuropeptide or a cytokine, it is evident it plays a key role in the communication between the nervous and immune systems:

- as a neuropeptide produced by neurons directly regulating nervous system functions;

- as a neuropeptide released by nerve endings in the lymphoid organs regulating immune functions;

- as a cytokine produced by $\mathrm{T}$ cells that regulates the immune system functions in an autocrine/ paracrine way;

- as a cytokine released from T cells that controls nervous system.

This study was supported by extramural grants from the Instituto de Salud Carlos III, Fondo de Investigacion Sanitaria, Spanish Ministry of Health (PI 030359 to D.P and PI 030526 to M.D.), from La Caixa Foundation (NE-03-009 to M.D) and from the European Union 6th Framework Grants Program (FP6-006380 to D.P).

\section{REFERENCES}

1. de Wied, D. (1974) Pituitary peptides and behaviour: influence on motivational, learning and memory processes. Excerpta Med. Int. Congr. Ser. 359, 653-658

2. Kastin, A. J. (2000) What is a neuropeptide? Trends Neurosci. 23, 113-114

3. Strand, F. L. (1999) Neuropeptides: Regulators of Physiological Processes, MIT Press, Cambridge, MA

4. Iversen, L. (1999) Neuopeptides: regulators of physiological processes. Trends Neurosci. 22, 482

5. Piper, P. J., Said, S. I., and Vane, J. R. (1970) Effects on smooth muscle preparations of unidentified vasoactive peptides from intestine and lung. Nature (London) 225, 1144-1146

6. Said, S., and Mutt, V. (1970) Polypeptide with broad biological activity: isolation from small intestine. Science 169, 1217-1218

7. Henning, R. J., and Sawmiller, D. R. (2001) Vasoactive intestinal peptide: cardiovascular effects. Cardiovasc. Res. 49, 27-37

8. Petkov, V., Mosgoeller, W., Ziesche, R., Raderer, M., Stiebellehner, L., Vonbank, K., Funk, G.-C., Hamilton, G., Novotny, C., Burian, B., et al. (2003) Vasoactive intestinal peptide as a new drug for treatment of primary pulmonary hypertension. J. Clin. Invest. 111, 1339-1346
9. Colwell, C. S., Michel, S., Itri, J., Rodriguez, W., Tam, J., Lelievre, V., Hu, Z., Liu, X., and Waschek, J. A. (2003) Disrupted circadian rhythms in VIP and PHI deficient mice. Am. J. Physiol. 285, R939-R949

10. Ganea, D., and Delgado, M. (2002) Vasoactive intestinal peptide (VIP) and pituitary adenylate cyclase-activating polypeptide (PACAP) as modulators of both innate and adaptive immunity. Crit. Rev. Oral. Biol. Med. 13, 229-237

11. Delgado, M. (2003) VIP: a very important peptide in T helper differentiation. Trends Immunol. 24, 221-224

12. Pozo, D. (2003) VIP- and PACAP-mediated immunomodulation as prospective therapeutic tools. Trends Mol. Med. 9, 211-217

13. Delgado, M., Abad, C., Martinez, C., Leceta, J., and Gomariz, R. P. (2001) Vasoactive intestinal peptide prevents experimental arthritis by downregulating both autoimmune and inflammatory components of the disease. Nat. Med. 7, 563-568

14. Grimm, M. C., Newman, R., Hassim, Z., Cuan, N., Connor, S. J., Le, Y., Wang, J. M., Oppenheim, J. J., and Lloyd, A. R. (2003) Cutting edge: vasoactive intestinal peptide acts as a potent suppressor of inflammation in vivo by trans-deactivating chemokine receptors. J. Immunol. 171, 4990-4994

15. Delgado, M., and Ganea, D. (2001) Cutting edge: is vasoactive intestinal peptide a type 2 cytokine? J. Immunol. 166, 2907-2912

16. Delgado, M., Leceta, J., and Ganea, D. (1999) Vasoactive intestinal peptide and pituitary adenylate cyclase-activating polypeptide stimulate the induction of Th2 responses by upregulating B7.2 expression. J. Immunol. 163, 3629-3635

17. Delgado, M., Reduta, A., Sharma, V., and Ganea, D. (2004) VIP/PACAP oppositely affects immature and mature dendritic cell expression of CD80/CD86 and the stimulatory activity for CD4+ T cells. J. Leukoc. Biol. 75, 1122-1130

18. Voice, J. K., Dorsam, G., Lee, H., Kong, Y., and Goetzl, E. J. (2001) Allergic diathesis in transgenic mice with constitutive T cell expression of inducible vasoactive intestinal peptide receptor. FASEB J. 15, 2489-2496

19. Goetzl, E. J., Voice, J. K., Shen, S., Dorsam, G., Kong, Y., West, K. M., Morrison, C. F., and Harmar, A. J. (2001) Enhanced delayed-type hypersensitivity and diminished immediate-type hypersensitivity in mice lacking the inducible VPAC2 receptor for vasoactive intestinal peptide. Proc. Natl. Acad. Sci. USA 98, 13854-13859

20. Guerrero, J., Prieto, J., Elorza, F., Ramirez, R., and Goberna, R. (1981) Interaction of vasoactive intestinal peptide with human blood mononuclear cells. Mol. Cell. Endocrinol. 21, 151-160

21. Voice, J. K., Grinninger, C., Kong, Y., Bangale, Y., Paul, S., and Goetzl, E. J. (2003) Roles of vasoactive intestinal peptide (VIP) in the expression of different immune phenotypes by wild-type mice and T cell-targeted type II VIP receptor transgenic mice. J. Immunol. 170, 308-314

22. Gozes, I., and Furman, S. (2003) VIP and drug design. Curr. Pharm. Des. 9, 483-494

23. Netea, M. G., van der Meer, J. W., van Deuren, M., and Kullberg, B. J. (2003) Proinflammatory cytokines and sepsis syndrome: not enough, or too much of a good thing? Trends Immunol. 24, 254-258

24. Delgado, M., Pozo, D., Martinez, C., Leceta, J., Calvo, J., Ganea, D., and Gomariz, R. (1999) Vasoactive intestinal peptide and pituitary adenylate cyclase activating polypeptide inhibit endotoxin-induced TNF- $\alpha$ production by macrophages: in vitro and in vivo studies. J. Immunol. 162, 2358-2367

25. Gomariz, R., Leceta, J., Martinez, C., Abad, C., Ganea, D., and Delgado, M. (2000) Anti-inflammatory actions of VIP/PACAP. Role in endotoxemia. Ann. N. Y. Acad. Sci. 921, 284-288

26. Delgado, M., and Ganea, D. (2001) Inhibition of endotoxininduced macrophage chemokine production by vasoactive intestinal peptide and pituitary adenylate cyclase-activating polypeptide: in vitro and in vivo. J. Immunol. 167, 966-975

27. Tuncel, N., Tore, F., Sahinturk, V., Ak, D., and Tuncel, M. (2000) Vasoactive intestinal peptide inhibits degranulation and changes granular content of mast cells: a potential therapeutic strategy in controlling septic shock. Peptides 21, 81-89

28. Williams, R. O. (2002) Therapeutic effect of vasoactive intestinal peptide in collagen-induced arthritis. Arthritis Rheum. 46, 271273

29. Feldmann, M., Brennan, F. M., and Maini, R. N. (1996) Role of cytokines in rheumatoid arthritis. Annu. Rev. Immunol. 14, $397-440$ 
30. Delgado, M., Abad, C., Martinez, C., Juarranz, G., Arranz, A., Gomariz, P., and Leceta, J. (2002) Vasoactive intestinal peptide in the immune system: potential therapeutic role in inflammatory and autoimmune diseases. J. Mol. Med. 80, 16-24

31. Strober, W., Fuss, I. J., and Blumberg, R. S. (2002) The immunology of mucosal models of inflammation. Annu. Rev. Immunol. 20, 495-549

32. Abad, C., Martinez, C., Juarranz, M. G., Arranz, A., Leceta, J., Delgado, M., and Gomariz, R. P. (2003) Therapeutic effects of vasoactive intestinal peptide in the trinitrobenzene sulfonic acid mice model of Crohn's disease. Gastroenterology 124, 961-971

33. Nguyen, M. D., Julien, J. P., and Rivest, S. (2002) Innate immunity: the missing link in neuroprotection and neurodegeneration? Nat. Rev. Neurosci. 3, 216-227

34. Delgado, M., and Ganea, D. (2003) Neuroprotective effect of vasoactive intestinal peptide (VIP) in a mouse model of Parkinson's disease by blocking microglial activation. FASEB J. 17, 944-946

35. Bramlett, H. M., and Dietrich, W. D. (2004) Pathophysiology of cerebral ischemia and brain trauma: similarities and differences. J. Cereb. Blood Flow Metab. 24, 133-150

36. Yoles, E., Hauben, E., Palgi, O., Agranov, E., Gothilf, A., Cohen, A., Kuchroo, V., Cohen, I. R., Weiner, H., and Schwartz, M. (2001) Protective autoimmunity is a physiological response to CNS trauma. J. Neurosci. 21, 3740-3748

37. Delgado, M., and Ganea, D. (2003) Vasoactive intestinal peptide prevents activated microglia-induced neurodegeneration under inflammatory conditions: potential therapeutic role in brain trauma. FASEB J. 17, 1922-1924

38. Gozes, I., Divinsky, I., Pilzer, I., Fridkin, M., Brenneman, D. E., and Spier, A. D. (2003) From vasoactive intestinal peptide (VIP) through activity-dependent neuroprotective protein (ADNP) to NAP: a view of neuroprotection and cell division. J. Mol. Neurosci. 20, 315-322

39. Laburthe, M., Couvineau, A., and Marie, J. C. (2002) VPAC receptors for VIP and PACAP. Receptors Channels 8, 137-153
40. Yung, S. L., Dela Cruz, F., Hamren, S., Zhu, J., Tsutsumi, M., Bloom, J. W., Caudle, M., Roczniak, S., Todd, T., Lemoine, L., et al. (2003) Generation of highly selective VPAC2 receptor agonists by high throughput mutagenesis of vasoactive intestinal peptide and pituitary adenylate cyclase-activating peptide. J. Biol. Chem. 278, 10273-10281

41. Bangale, Y., Karle, S., Planque, S., Zhou, Y. X., Taguchi, H., Nishiyama, Y., Li, L., Kalaga, R., and Paul, S. (2003) VIPase autoantibodies in Fas-defective mice and patients with autoimmune disease. FASEB J. 17, 628-635

42. Pozo, D., Delgado, M., Martinez, C., Gomariz, R., Guerrero, J., and Calvo, J. (1997) Functional characterization and mRNA expression of pituitary adenylate cyclase activating polypeptide (PACAP) type I receptors in rat peritoneal macrophages. Biochim. Biophys. Acta 1359, 250-262

43. Van Rampelbergh, J., Poloczek, P., Francoys, I., delporte, C., Winand, J., Robberecht, P., and Waelbroeck, M. (1997) The pituitary adenylate cyclase polypeptide (PACAP I) and VIP (PACAP II VIP ${ }_{1}$ ) receptors stimulate inositol phosphate synthesis in transfected $\mathrm{CHO}$ cells through interaction with different $\mathrm{G}$ proteins. Biochim. Biophys. Acta 135, 249-255

44. Christopoulos, A., Christopoulos, G., Morfis, M., Udawela, M., Laburthe, M., Couvineau, A., Kuwasako, K., Tilakaratne, N., and Sexton, P. M. (2003) Novel receptor partners and function of receptor activity-modifying proteins. J. Biol. Chem. 278, 32933297

45. McCulloch, D. A., MacKenzie, C. J., Johnson, M. S., Robertson, D. N., Holland, P. J., Ronaldson, E., Lutz, E. M., and Mitchell, R. (2002) Additional signals from VPAC/PAC family receptors. Biochem. Soc. Trans. 30, 441-446 